\title{
Nonperturbative and Parton Shower corrections in matched NLO-shower event generators
}

Samantha Katherine DOOLING*

(Deutsches Elektronen-Synchrotron ( DESY))

E-mail: samantha.dooling@desy.de

\section{Paolo Gunnellini}

(Deutsches Elektronen-Synchrotron ( DESY))

E-mail: paolo.gunnellini@desy.de

\section{Francesco Hautmann}

( Theoretical Physics Department, University of Oxford,

Physics and Astronomy, University of Sussex)

E-mail: hautmanethphys.ox.ac.uk

\section{Hannes Jung}

( Deutsches Elektronen-Synchrotron ( DESY),

Elementaire Deeltjes Fysica, Universiteit Antwerpen)

E-mail: hannes.jung@desy.de

\begin{abstract}
Comparisons of experimental data with theoretical predictions for collider processes containing hadronic jets rely on Shower Monte Carlo event generators to include corrections to perturbative calculations from hadronization, parton showering, multiple parton collisions. We examine current treatments of these corrections and propose alternative methods to take into account nonperturbative effects and parton showering in the context of next-to-leading-order (NLO) event generators. We point out sizeable parton-showering corrections to jet transverse energy spectra at high rapidity, and discuss kinematic shifts in longitudinal momentum distributions from initialstate showering.
\end{abstract}

XXI International Workshop on Deep-Inelastic Scattering and Related Subjects 22-26 April, 2013

Marseilles, France

\footnotetext{
*Speaker.
} 
In high energy physics the experimental data of jet production processes are compared to next-to-leading order (NLO) theory calculations to stringently test QCD. The predictions consist of perturbative QCD calculation and a correction to account for effects beyond perturbation theory. Shower Monte Carlo (SMC) event generators are used to estimate the correction factors to be applied to NLO theory calculations to account for nonperturbative effects, due to multiparton interactions and hadronization. In this article we present a new approach [1] treating the nonperturbative corrections in the context of matched NLO-shower MC generators.

At the LHC jets are measured over a much wider range than in previous collider experiments. The ATLAS [2] and CMS [3] Collaborations measured inclusive jets up to the TeV scale in jet transverse momentum and in rapidity up to 4.4 and 3.0, respectively. Comparisons of inclusive jet data and NLO $\times$ NP theory predictions show good agreement in the central region but show differences in the forward region. Motivated by this we studied the effect of the parton showers in the forward region and define a new correction [1], which accounts for the effect of soft parton emissions. In order to derive the nonperturbative and parton shower corrections with highest accuracy a NLO-MC generator is used to determine the hard scattering. The events are generated within the POWHEG BOX framework ${ }^{1}$ and are showered with the SMC PYTHIA6 [4] . In the POWHEG [5] method the hard matrix element is determined at NLO and is matched with the parton showers, which take into account higher order radiation contributions. In previous analysis [3] leading-order Monte Carlo (LO-MC) generators $[4,6]$ are used to estimate the nonperturbative correction, which is defined as the nominal generator setting including parton showers (ps), multiparton interaction (mpi) and hadronziation (had) divided by the LO hard process supplemented with parton showers

$$
K_{0}^{\mathrm{NP}}=N_{\mathrm{LO}-\mathrm{MC}}^{(\mathrm{ps}+\mathrm{mpi}+\mathrm{had})} / N_{\mathrm{LO}-\mathrm{MC}}^{(\mathrm{ps})}
$$

This is the most obvious way to define the nonperturbative correction with a LO-MC generator. However, when combing the NLO calculation with parton shower and hadronization, the real correction of the first parton emission is treated differently in the NLO calculation and the parton shower [1]. We propose an alternative method which uses a NLO-MC event generator to determine the corrections. Furthermore, we define an additional correction factor to the fixed-order calculation due to parton showering effects. The parton shower correction is new and it singles out contributions due to higher order parton emissions. The corrections are defined as [1]

$$
\begin{gathered}
K^{\mathrm{NP}}=N_{\mathrm{NLO}-\mathrm{MC}}^{(\mathrm{ps}+\mathrm{mpi}+\mathrm{had})} / N_{\mathrm{NLO}-\mathrm{MC}}^{(\mathrm{ps})}, \\
K^{\mathrm{PS}}=N_{\mathrm{NLO}-\mathrm{MC}}^{(\mathrm{ps})} / N_{\mathrm{NLO}-\mathrm{MC}}^{(0)},
\end{gathered}
$$

where $N_{\mathrm{NLO}-\mathrm{MC}}^{(0)}$ is obtained by switching of all components beyond NLO in the MC simulation. The difference in the nonperturbative corrections obtained by a LO, eq. (1), or a NLO, eq. (2), calculation is due to the matching of multiple parton interactions to the NLO calculation. The multi parton interaction $p_{\mathrm{T}}$ scale is smaller than the transverse momentum of the hard process, which is defined by the average $p_{\mathrm{T}}$ of the hard partons in the process. Thus in LO and NLO the hard scale is different, which leads to a numerical difference in the nonperturbative correction factor. In fig. (1) the comparison of the nonperturbative correction obtained by the LO generator PYTHIA

\footnotetext{
${ }^{1}$ http://powhegbox.mib.infn.it/
} 

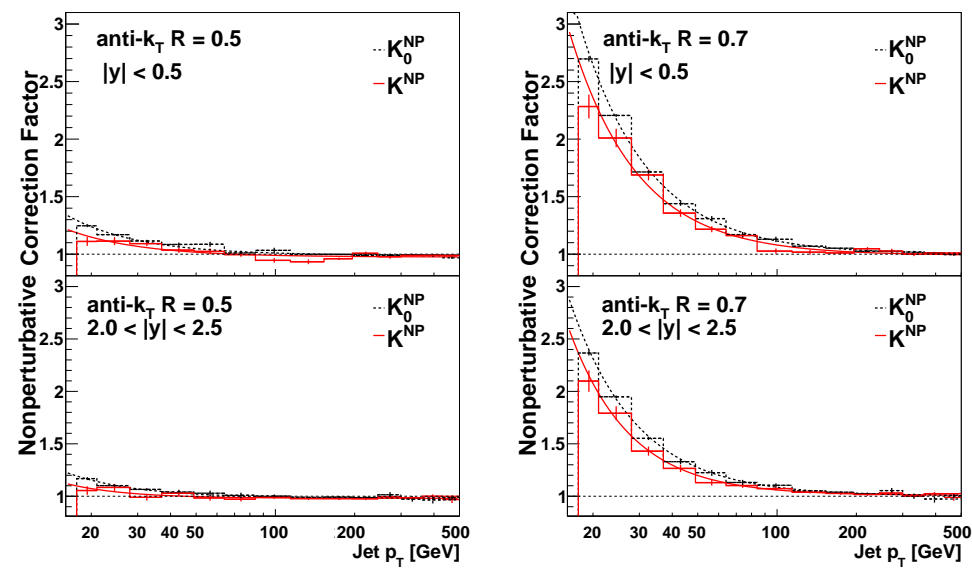

Figure 1: The nonperturbative correction factors [1] to jet transverse momentum distributions obtained using PYTHIA and POWHEG+PYTHIA. The jets are defined by the anti- $k_{\mathrm{T}}$ algorithm for two different jet sizes and are compared in the central and forward rapidity range.

from eq. (1) and the matched NLO generator POWHEG+PYTHIA from eq. (2) is shown. At small transverse momentum the nonpertubative correction shows a non-negligible effect and a difference between LO and NLO is observed. With increasing $p_{\mathrm{T}}$ the nonperturbative corrections $K_{0}^{\mathrm{NP}}$ and $K^{\mathrm{NP}}$ both approach one. The correction factor to account for parton emission is shown in fig. (3). The parton shower correction depends on $p_{\mathrm{T}}$ and rapidity. Especially in the forward region the correction gives a finite effect also at high $p_{\mathrm{T}}$. In general the effect of parton shower is largest at large $y$. In fig. (2) the effect of initial- and final-state showers are considered separately. The initial-state radiation is mainly contributing at low $p_{\mathrm{T}}$, while the final-state radiation is contributing over the whole $p_{\mathrm{T}}$ range. It has to be noted here that the effects of the initial-state radiation and final-state radiation are interconnected. The combined effect of parton shower cannot be obtained by simply adding up the two contributions. In addition the effect of parton showers can become smaller than the individual contributions from initial- and final-state showers at large $p_{\mathrm{T}}$, coming from migration effects in $p_{\mathrm{T}}$ and $y$.

Kinematic effects in the longitudinal momentum fraction due to initial-state showering are considered in [7]. Motivated by this we studied the influence of the parton shower to the longitudinal momentum fraction $x$ of the partons at high rapidities. In general the cross section of a perturbative process is factorized in the hard subprocess and the parton density functions of the incoming partons with energy momentum fraction $x$. The momentum $k_{j}^{(0)}=x_{j} p_{j}$ is treated collinear with the beam hadron and the parton is on mass-shell. However when applying a shower algorithm, the initial- and final-state partons emit QCD radiation and are no longer collinear with the beam hadron $k_{j} \neq x_{j} p_{j}$. Their transverse momentum has to be compensated by a change in the kinematics of the hard subprocess. This implies a reshuffling in the longitudinal momentum fraction $x_{j}$ of the partons, due to energy momentum conservation. In the case of inclusive jet production with $p_{\mathrm{T}}>20$ $\mathrm{GeV}$ in rapidities $|y|<2.5$, fig. (4) shows the distribution as a function of the parton longitudinal momentum fraction $x$ before and after parton showering. One can see that the longitudinal momentum fraction is shifted to higher values of $x$ when including QCD radiation. This effect is negligible 

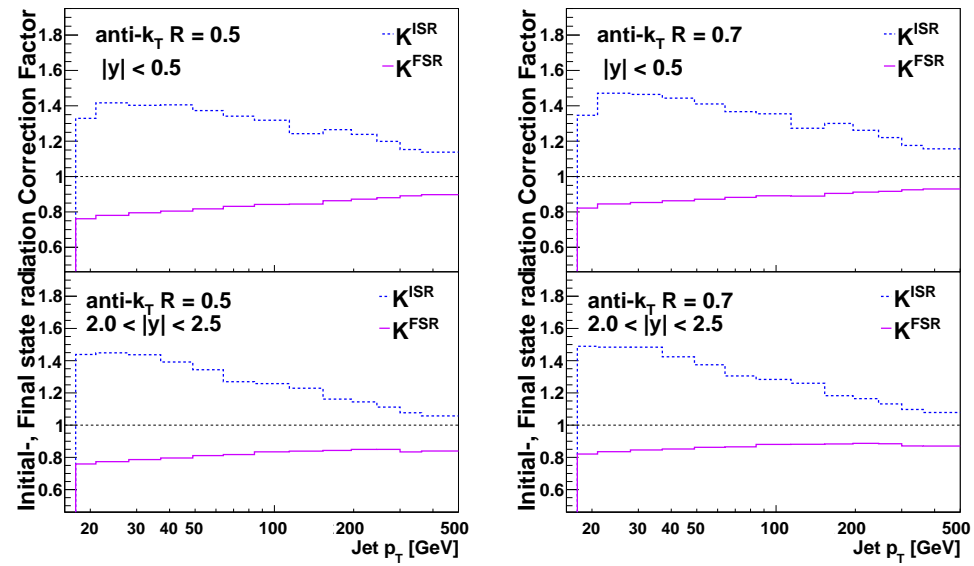

Figure 2: The intial- and final-state parton shower effects [1] to jet transverse momentum distributions, obtained from POWHEG+PYTHIA. The jets are defined by the anti- $k_{\mathrm{T}}$ algorithm with two different jet radia and compared in central and forward rapidities.
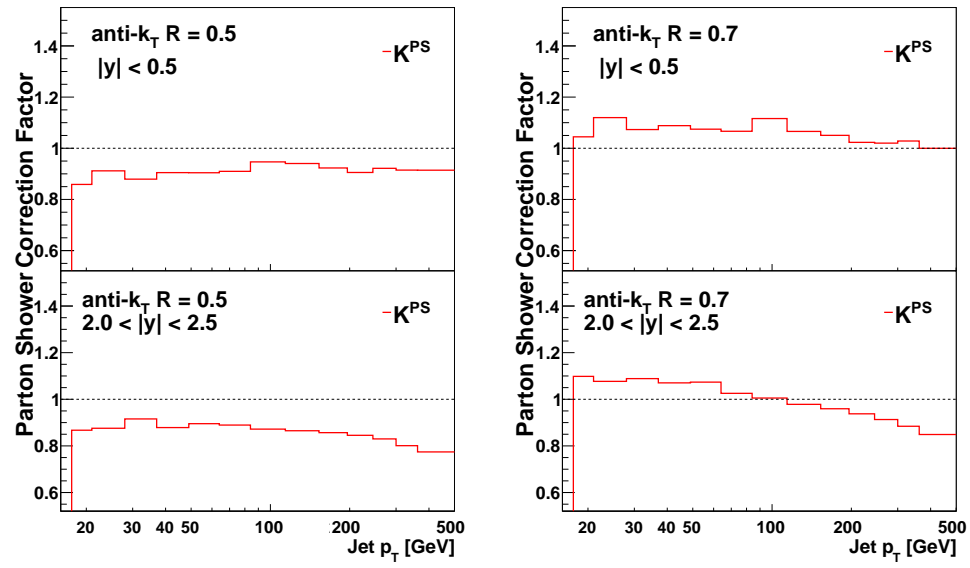

Figure 3: The parton shower correction factors [1] to jet transverse momentum distributions, obtained from POWHEG+PYTHIA. The jets are defined by the anti- $k_{\mathrm{T}}$ algorithm with two different jet radia and compared in central and forward rapidities.

for central rapidities but becomes important at $|y|>1.5$. The momentum reshuffling is done after the evaluation of the parton density functions (pdfs) and therefore can effect the determination of the pdfs. The effect of the kinematic shift is common for different production processes. In [1] further results for massive final states relevant at the LHC are studied.

To summarize, the use of matched NLO-MC generators can affect the comparison of theory predictions to inclusive jet data. The "new" parton shower corrections are significant over the whole $p_{\mathrm{T}}$ range, but mostly at large rapidities. The dependence on $p_{\mathrm{T}}$ and $y$ can influence the shape of parton distribution functions. Furthermore, combining the collinear approximation and energy momentum constrains implies kinematic shifts in longitudinal momentum distributions. This effect is 

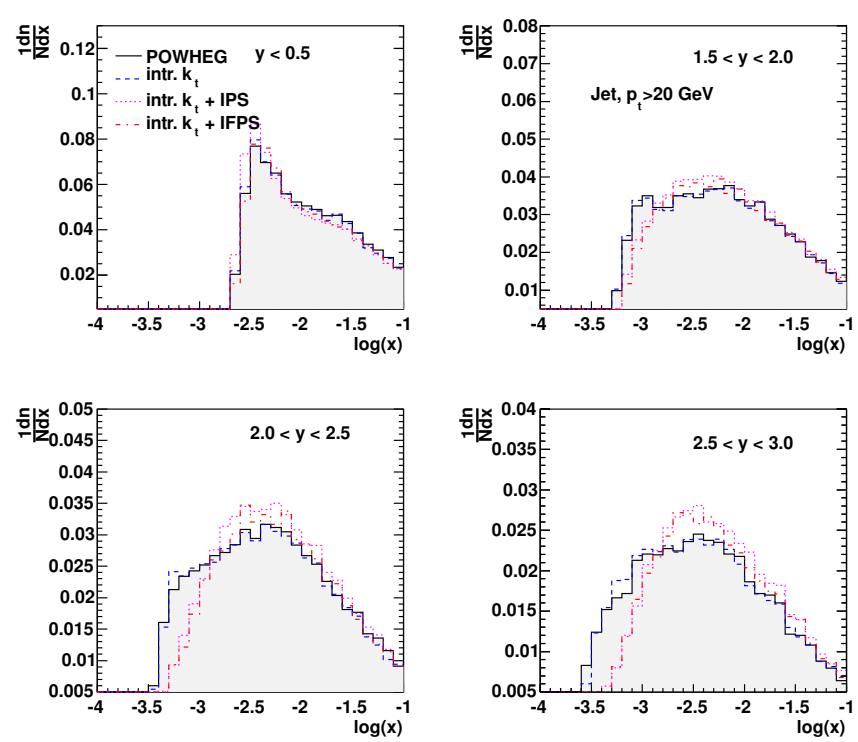

Figure 4: Distributions in the parton longitudinal momentum fraction $x$ before (POWHEG) and after parton showering (POWHEG+PS) [1], for inclusive jet production at different rapidities for jets defined by the anti$k_{\mathrm{T}}$ jet algorithm with $R=0.5$. Shown is the effect of intrinsic $k_{\mathrm{T}}$, initial- (IPS) and initial+final-state (IFPS) parton shower.

negligible at central rapidities but is mostly pronounced at rapidities $|y|>1.5$. We want to stress that the proper treatment of nonperturbative and parton shower corrections by using matched NLOMC generators can influence fits for parton distribution functions and $\alpha_{\mathrm{S}}$ extraction using inclusive jet data.

\section{Acknowledgements}

We are grateful to the DIS organizers and staff for the invitation to an exciting conference. We want to thank all DIS participants for a nice atmosphere and interesting discussions.

\section{References}

[1] S. Dooling, P. Gunnellini, F. Hautmann and H. Jung, arXiv:1212.6164 [hep-ph].

[2] ATLAS Coll. (G. Aad et al.), Phys. Rev. D 86 (2012) 014022.

[3] CMS Coll. (S. Chatrchyan et al.), Phys. Rev. Lett. 107 (2011) 132001; arXiv:1106.0208 [hep-ex].

[4] T. Sjöstrand, S. Mrenna and P. Skands, JHEP 0605 (2006) 026.

[5] S. Alioli et al., JHEP 1104 (2011) 081.

[6] G. Corcella et al., JHEP 0101 (2001) 010 arXiv:hep-ph/0210213.

[7] F. Hautmann and H. Jung, Eur. Phys. J. C 72 (2012) 2254. 